\title{
Peroral endoscopic myotomy and simultaneous endoscopic diverticuloseptotomy in a case of achalasia with diverticula
}

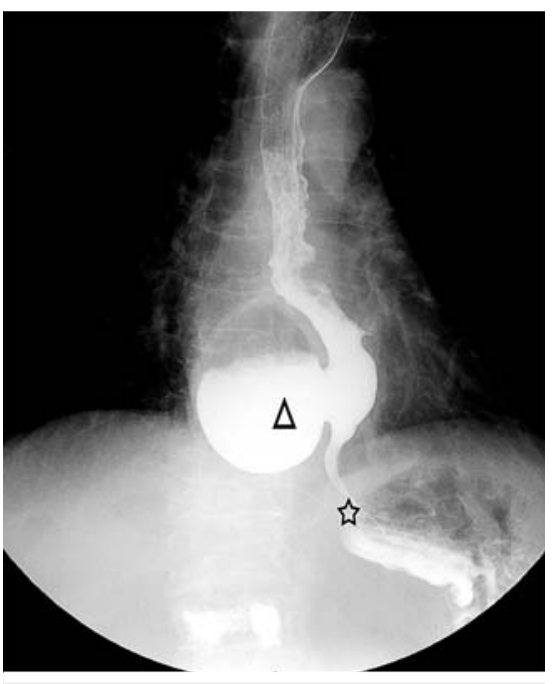

- Fig. 1 Contrast esophagogram before peroral endoscopic myotomy shows a large epiphrenic diverticulum (black triangle) on the right anterolateral esophageal wall. A thin streak of contrast medium is visible across the lower esophageal sphincter (black star).

A 58-year-old man with symptoms of dysphagia and regurgitation was admitted for treatment. Evaluation with endoscopy and contrast esophagogram revealed achalasia cardia with a small mid-esophageal diverticulum and a large epiphrenic diverticulum ( $\triangleright$ Fig. 1, $\triangleright$ Fig.2). Peroral endoscopic myotomy (POEM) and a simultaneous endoscopic diverticuloseptotomy were performed in this case ( Video 1). Firstly, submucosal injection and mucosal incision were made at about $5 \mathrm{~cm}$ above the epiphrenic diverticulum. Secondly, a submucosal tunnel was created pointing toward the diverticular septum and extended on both sides of the septum, i.e., the diverticular and esophageal lumen side ( $\triangleright$ Fig.3). Thirdly, complete myotomies of the muscle layer of the diverticular septum and the esophagus were performed separately. Due to technical difficulty, a small mucosal perforation occurred at the most narrow, twisted, and spasmodic part of the distal
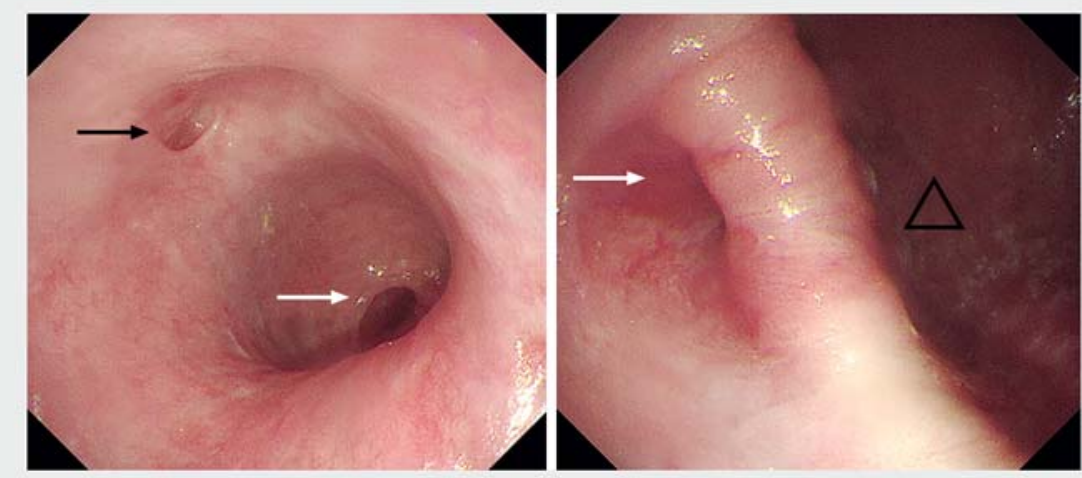

- Fig. 2 Endoscopic images show a small mid-esophageal diverticulum (black arrow) and a large epiphrenic diverticulum (black triangle). White arrows show the entrance of the narrow lumen of distal esophagus.
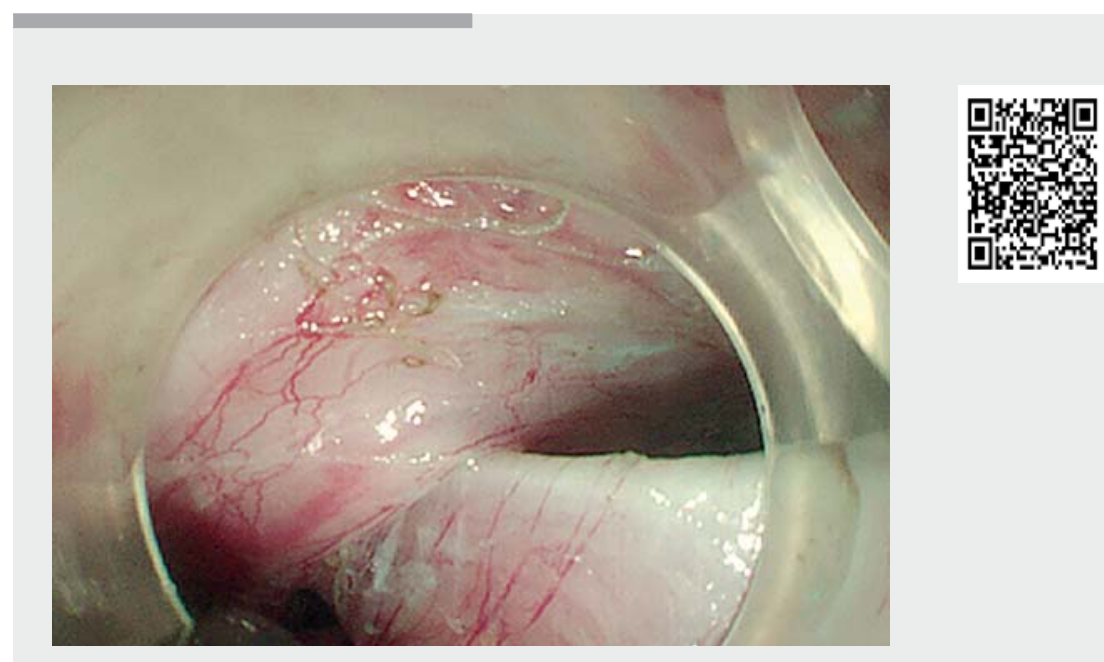

$\checkmark$ Video 1 Peroral endoscopic myotomy and simultaneous endoscopic diverticuloseptotomy in a case of achalasia with diverticula.

esophagus ( $\mathbf{F i g} \mathbf{4}$ ). In the final step, the small mucosal injury and the entry of the tunnel were closed with endoclips. An X-ray contrast study 5 days after POEM revealed a free flow of contrast medium across the gastroesophageal junction and a collapsed epiphrenic diverticulum ( Fig.5). There was substantial improvement in the patient's clinical symptoms as well. At the 3-month follow-up, the patient reported complete resolution of dysphagia.

POEM has been introduced for achalasia treatment as a less invasive alternative to laparoscopic Heller myotomy [1]. However, a few patients with achalasia have a co-existing large epiphrenic diverticulum, which may cause technical difficulties and increase the rates of procedure-related adverse events [2,3]. In our 


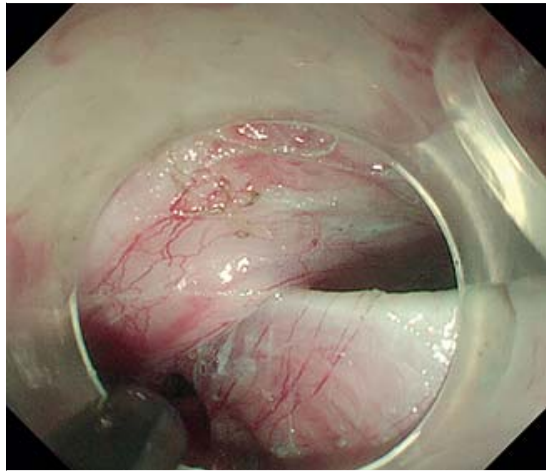

- Fig. 3 A submucosal tunnel was created pointing toward the diverticular septum and extended on both sides of the septum.

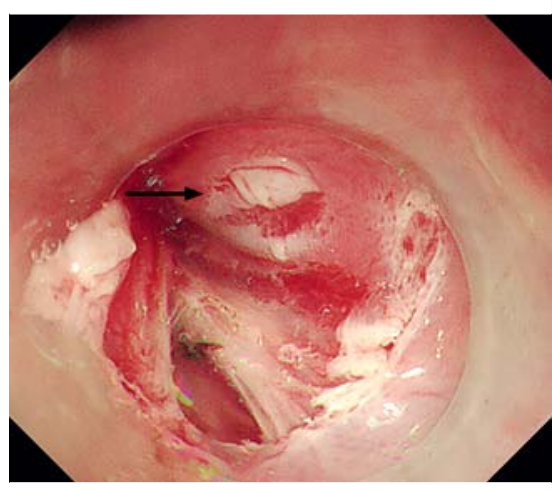

- Fig. 4 A small mucosal injury occurred at the most narrow, twisted, and spasmodic part of the distal esophagus during peroral endoscopic myotomy (black arrow).

case, a small esophageal mucosal injury occurred during POEM, which was completely sealed by an endoclip without any postoperative complications. Classically, a diverticulum of the middle esophagus is classified as a Rokitansky diverticulum, and it rarely attains an appreciable size or produces any symptoms [4]. We did not treat the small midesophageal diverticulum in this case.

Endoscopy_UCTN_Code_TTT_1AO_2AM

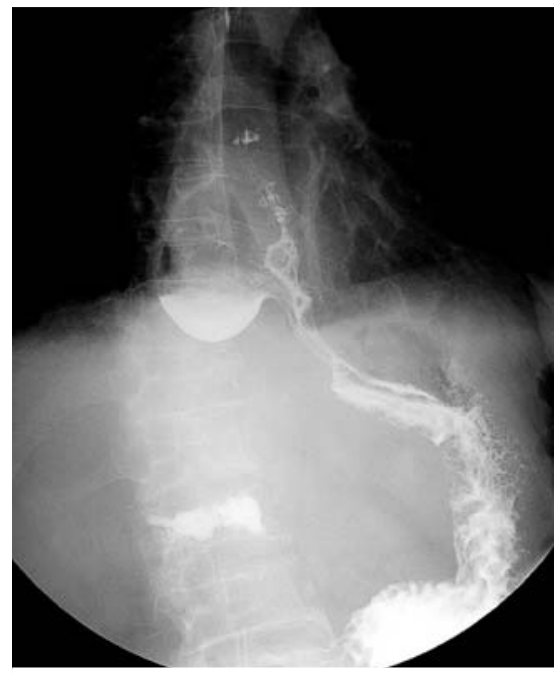

- Fig. 5 Contrast esophagogram 5 days after peroral endoscopic myotomy shows quick passage of contrast medium across the gastroesophageal junction into the stomach; the previously large diverticulum has collapsed.

\section{Competing interests}

None

\section{The authors}

Wen Li ${ }^{1}$, Fang Liu ${ }^{1}$, Liang $\mathbf{W u}^{2}$

1 Department of Gastroenterology and Hepatology, The First Medical Center of Chinese PLA General Hospital, Beijing, China

2 International Center for Diagnosis and Treatment of Liver Diseases, The Fifth Medical Center of Chinese PLA General Hospital, Beijing, China

\section{Corresponding author}

\section{Liang Wu}

International Center for Diagnosis and

Treatment of Liver Diseases, The Fifth Medical Center of Chinese PLA General Hospital, Beijing, 100039, China Fax: +86-10-55499107 wuliangdoc@163.com

\section{References}

[1] Zaninotto G, Leusink A, Markar SR. Management of achalasia in 2019. Curr Opin Gastroenterol 2019. doi:10.1097/ MOG.0000000000000544

[2] Bechara R, Woo M, Hookey L et al. Peroral endoscopic myotomy (POEM) for complex achalasia and the POEM difficulty score. Dig Endosc 2019; 31: 148-155

[3] Li HK, Linghu EQ. New endoscopic classification of achalasia for selection of candidates for peroral endoscopic myotomy. World J Gastroenterol 2013; 19: 556-560

[4] Sato H, Takeuchi M, Hashimoto S et al. Esophageal diverticulum: New perspectives in the era of minimally invasive endoscopic treatment. World J Gastroenterol 2019; 25: 1457-1464

\section{Bibliography}

DOI https://doi.org/10.1055/a-1059-9322

Published online: 2.12.2019

Endoscopy 2020; 52: E168-E169

(c) Georg Thieme Verlag KG

Stuttgart - New York

ISSN 0013-726X

\section{ENDOSCOPY E-VIDEOS}

https://eref.thieme.de/e-videos

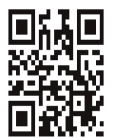

Endoscopy E-Videos is a free access online section, reporting on interesting cases and new techniques in gastroenterological endoscopy. All papers include a high quality video and all contributions are freely accessible online.

This section has its own submission website at https://mc.manuscriptcentral.com/e-videos 